\title{
Superconducting Gap within a Modified Interlayer Tunneling Model
}

\author{
Biplab Chattopadhyay ${ }^{\star}$ and A. N. $\operatorname{Das}^{\dagger}$ \\ Saha Institute of Nuclear Physics, 1/AF Bidhannagar, Calcutta - 700 064, INDIA
}

\begin{abstract}
A modified version of the interlayer tunneling model, including interlayer single particle hopping (ISPH), is considered as a phenomenological model to describe cuprate superconductors. The effective ISPH $\left(t_{\perp}^{\mathrm{eff}}\right)$ is taken along with a probability factor $P$, that involves the normal state pseudogap $\left(E_{g}\right)$. This makes $t_{\perp}^{\text {eff }}$ to mimic experimental observations that, ISPH is small in the underdoped regime and increases towards overdoping. Within the modified model, we establish the absence of bilayer splitting as observed in case of layered cuprates. Transition temperature $\left(T_{c}\right)$ and the superconducting gap are calculated. A match, to the T-dependent superconducting gap data from experiment, is obtained and high values of the ratio of the superconducting gap to $T_{c}$ are recovered. Depending on the values of $E_{g}, T_{c}$ as a function of interlayer coupling shows mixed behaviour. This is a prediction and can be checked further.
\end{abstract}

PACS numbers: 74.20.-z, 74.62.Dh, 74.25.Bt

Keywords: Interlayer single particle hopping, Bilayer splitting, Superconducting gap.

Physics Letters A (to appear).

^email: biplab@cmp.saha.ernet.in; †email: atin@cmp.saha.ernet.in 
Surge of research activity on high temperature superconductors (HTS) in the last few years, have clearly established that, the HTS have unusual normal as well as superconducting state properties different from those of the conventional BCS superconductors. In the superconducting state, the issues related to the superconducting gap, such as the symmetry of the gap, ratio of the gap to $T_{c}$ and the temperature variation of the gap, are worth addressing. There is growing evidence that the gap of the HTS has $d_{x^{2}-y^{2}}$ symmetry with line nodes [1, 2], in contrast to the s-wave gap of the BCS superconductors. Similarly, temperature dependence of the superconducting gap and the value of the gap ratio, as observed in experiments [3, 4], are quite different from those of the conventional superconductors.

Endeavour to explain various unusual properties of HTS, resulted in the introduction of a number of phenomenological models which are commonly characterized by a BCS like gap equation together with well defined quasiparticles in the superconducting state. One such model is the interlayer tunneling model originally proposed by P. W. Anderson [5]. The interlayer tunneling (ILT) model describes bilayer cuprates where interlayer single particle hopping (ISPH) is taken to be inhibited due to correlation effects, but tunneling of Cooper pairs between the layers is considered. This pair tunneling amplifies the pairing mechanism within a $\mathrm{CuO}_{2}$ layer and an increase in transition temperature $\left(T_{c}\right)$ results [6]. The ILT model could account for high $T_{c}$ observed in cuprate superconductors, but there are difficulties in recovering very high values of the gap ratio for realistic parameters as well as matching the experimental gap variation data within the model. At this point, a relevant question to ask is whether the introduction of ISPH necessary. As a matter of fact, in band structure calculations, the ISPH matrix elements are found to be substantial for bilayer cuprates [7]. In addition, experimental observations on sufficiently overdoped cuprates find metallic-like behaviour of $c$-axis resistivity and the presence of Drude peak in the $c$-axis optical conductivity [8, 9]. These features immediately imply that, in case of HTS the existence of ISPH cannot be ruled out at least in the overdoped regime. 
Motivated by the experimental observations [8, 9], band structure calculations [7] and the discrepancies reflected in the properties involving the superconducting gap within the ILT model, we consider ISPH along with pair hopping to modify the ILT model. For underdoped cuprates, the c-axis resistivity shows semiconducting behavior and Drude peak is absent in the optical conductivity along the c-axis. These are signatures of the absence or marginal presence of ISPH in the underdoped systems and hence, it is considered along with a probability factor $P$ such that the effective ISPH is very small for underdoping and attains significance for overdoped systems. Pair hopping involves the particles which are not taking part in the ISPH. Hence, the probability for pair hopping is defined [10, 11] as $(1-P)^{2}$.

By a detailed study of the density of states within the modified ILT model, we address the issue of bilayer splitting for layered cuprates. Obtaining the gap equation within the mean-field approximation we calculate the superconducting gap as a function of temperature, and at optimal doping we study the variation of $T_{c}$ with the interlayer coupling. Principal results from our calculations are as follows. We demonstrate the absence of bilayer splitting within the modified model validating the inclusion of ISPH. Variation of $T_{c}$ with interlayer coupling is different for different values of $E_{g}$. Temperature variation of the gap from our calculations has an excellant match to the experimental data [4] and ratio of the superconducting gap to $T_{c}$ has high values as in experiments [3].

The Model: The Hamiltonian of the coupled bilayer complex [10, 11] is written as

$$
\begin{aligned}
H & =\sum_{i, \mathbf{k}, \sigma}\left(\epsilon_{\mathbf{k}}-\mu\right) c_{\mathbf{k} \sigma}^{(i) \dagger} c_{\mathbf{k} \sigma}^{(i)}-\sum_{i, \mathbf{k}, \mathbf{k}^{\prime}}\left[V_{\mathbf{k}, \mathbf{k}^{\prime}} c_{\mathbf{k} \uparrow}^{(i) \dagger} c_{-\mathbf{k} \downarrow}^{(i) \dagger} c_{-\mathbf{k}^{\prime} \downarrow}^{(i)} c_{\mathbf{k}^{\prime} \uparrow}^{(i)}+h . c\right] \\
& -\sum_{i \neq j, \mathbf{k}}\left[T_{p}^{\mathrm{eff}}(\mathbf{k}) c_{\mathbf{k} \uparrow}^{(i) \dagger} c_{-\mathbf{k} \downarrow}^{(i) \dagger} c_{-\mathbf{k} \downarrow}^{(j)} c_{\mathbf{k} \uparrow}^{(j)}+h . c\right]+\sum_{i \neq j, \mathbf{k}, \sigma}\left[t_{\perp}^{\mathrm{eff}}(\mathbf{k}) c_{\mathbf{k} \sigma}^{(i) \dagger} c_{\mathbf{k} \sigma}^{(j)}+h . c\right]
\end{aligned}
$$

This is similar to the one considered by Anderson and coworkers [12], barring the last term accounting for the interlayer single particle hopping. The operator $c_{\mathbf{k} \sigma}^{(i) \dagger}\left(c_{\mathbf{k} \sigma}^{(i)}\right)$ creates (annihilates) a fermion in the $i$-th layer $(i=1,2)$ with momentum $\mathbf{k}$ and spin index $\sigma$. Here $V_{\mathbf{k}, \mathbf{k}^{\prime}}$ is the interaction potential forming Cooper pairs in the $a b$-plane and $\mu$ is the chemical potential. For the $a b$-plane band dispersion $\epsilon_{k}$, we use the realistic band structure 
obtained from a six parameter tight binding fit $\left[t_{0}, t_{1}, t_{2}, t_{3}, t_{4}, t_{5}\right]=[0.131,-0.149,0.041,-$ 0.013,-0.014,0.013] eV to the ARPES data [13] on Bi2212. This six parameter band dispersion, used elsewhere [14], shows flat bands in the Brillouin zone and the corresponding density of states (DOS) has a power law singularity known as extended van Hove singularity. This is characteristic to the high- $T_{c}$ cuprates [15]. The effective ISPH matrix element is taken to be $t_{\perp}^{\text {eff }}(\mathbf{k})=t_{\perp}^{b}(\mathbf{k}) P$, where $t_{\perp}^{b}(\mathbf{k})=t_{\perp}\left(\left(\cos k_{x} a-\cos k_{y} a\right) / 2\right)^{2}$ is the $\mathbf{k}$-dependent ISPH adapted from the band structure calculations [7] with $t_{\perp}$ being the bare ISPH matrix element ( $a$ is the lattice constant) and $P$ is a probability factor determined by the normal state pseudogap found recently in layered cuprates [16]. Particles that are not taking part in the ISPH, are available for pair tunneling and complementary probability for each partner of a pair is $(1-P)$. This fixes the tunneling probability of a pair as $(1-P)^{2}$, and following Anderson [6] we take the effective pair tunneling $T_{p}^{\mathrm{eff}}(\mathbf{k})=\left[\left(t_{\perp}^{b}(\mathbf{k})\right)^{2} /\left|t_{1}\right|\right](1-P)^{2}$, where $t_{1}$ is the nearest neighbour hopping matrix element of the $a b$-plane band dispersion 13 .

Interlayer Single Particle Hopping Probability and the Normal State Pseudogap: In experiments on layered materials, an important measurable quantity is the $c$-axis resistivity which, when studied as a function of doping $(\delta)$, provides usefull information about the strength of the effective single particle hopping between the layers. For layered cuprates, the $c$-axis resistivity shows semiconducting behaviour in the underdoped regime [17] and has metallic-like temperature dependence in the heavily overdoped regime 8, 9]. This has direct bearing on the ISPH and implies that the effective ISPH $\left(t_{\perp}^{\mathrm{eff}}\right)$ is strong enough for overdoping, decreases gradually through optimal doping and becomes weaker towards underdoping. The probability factor $P$, buried in $t_{\perp}^{\text {eff }}$ could account for this doping dependence, when suitably expressed in terms of the temperature and the normal state pseudogap $E_{g}$ of cuprate superconductors. The magnitude of $E_{g}$ is found to be vanishingly small for overdoping and it increases towards underdoping 18. Previously, we considered an exponential form [10, 11] for the probability factor, $P=\exp \left(-E_{g} / T\right)$ and found that the experimental gap variation data, in the low temperature region, were 
not precisely recoverable. We also noticed that a linear $T$-dependent form for $P$ could significantly improve the matching of experimental data. Thus, here we consider a linear (low-temperature) $T$-dependent form (for $E_{g} \gg T$ ) of the probability factor as

$$
P=\frac{T}{E_{g}+T \mathrm{e}^{-E_{g} / T}}
$$

where $T=\beta^{-1}$ with the Boltzmann constant $k_{B}$ set to unity. Clearly, $P \rightarrow 1$ for $E_{g} \rightarrow 0$ (heavily overdoped situation) and $P \rightarrow 0$ as $E_{g} \rightarrow \infty$ (underdoped situation). Thus $P$, as in $\mathrm{Eq}(2)$, consistently mimics the observable doping dependence of the effective ISPH. Though a concrete justification of $\mathrm{Eq}(2)$ is not possible at present, we present plausible arguments in favour of the linear $T$-dependence of $P$ in connection with the RVB model.

Within the RVB framework, where spin-charge separation yields spinon and holon quasiparticles, Anderson argued that a real hole can come into being in a layer only when a holon combines with a spinon and this hole can then hop to another layer. Thus, within RVB model, $c$-axis transport is proportional to the spinon density. Since, in the RVB model, the spinon density is proportional to $T$, hence the linear $T$-dependence of $c$-axis transport follows. In calculations, we incorporate the momentum dependence of the normal state pseudogap, which is found to be of $d_{x^{2}-y^{2}}$ symmetry [16], and we take $E_{g}(\mathbf{k})=E_{g}\left|\cos k_{x} a-\cos k_{y} a\right|$.

Self Consistent Equations for the Superconducting Gap and the Chemical Potential:

Within the Hartree-Fock approximation, self consistent equations for the superconducting gap $\Delta_{\mathbf{k}}$ and the chemical potential $\mu$ (fixing the average number of electrons per site $n=1-\delta$ ) are derived for the modified model under consideration. Decoupling of the four fermion terms in the Hamiltonian of $\mathrm{Eq}(1)$ gives

$$
\begin{aligned}
H & =\sum_{i, \mathbf{k}, \sigma}\left(\epsilon_{k}-\mu\right) c_{\mathbf{k} \sigma}^{(i) \dagger} c_{k \sigma}^{(i)}-\sum_{i, \mathbf{k}}\left[\Delta_{\mathbf{k}} c_{\mathbf{k} \uparrow}^{(i) \dagger} c_{-\mathbf{k} \downarrow}^{(i) \dagger}+h . c\right] \\
& +\sum_{i \neq j, \mathbf{k}, \sigma}\left[t_{\perp}^{\mathrm{eff}}(\mathbf{k}) c_{\mathbf{k} \sigma}^{(i) \dagger} c_{\mathbf{k} \sigma}^{(j)}+h . c\right]
\end{aligned}
$$


where the $\mathbf{k}$-dependent superconducting gap is

$$
\Delta_{\mathbf{k}}=\Delta_{i, \mathbf{k}}=\sum_{\mathbf{k}^{\prime}} V_{\mathbf{k}, \mathbf{k}^{\prime}}\left\langle c_{-\mathbf{k}^{\prime} \downarrow}^{(i)} c_{\mathbf{k}^{\prime} \uparrow}^{(i)}\right\rangle+T_{p}^{\mathrm{eff}}(\mathbf{k})\left\langle c_{-\mathbf{k} \downarrow}^{(j)} c_{\mathbf{k} \uparrow}^{(j)}\right\rangle
$$

which includes pairing in one layer as well as a contribution from pairing in the other layer owing to the pair tunneling mechanism. Since the in-plane pairing is identical in both the layers, we suppress labelling the gap parameter by layer index. Coherent single particle hopping between the layers produces two quasiparticle bands

$$
E_{\mathbf{k}}^{-}=\sqrt{\left\{\epsilon_{\mathbf{k}}-\mu-t_{\perp}^{\mathrm{eff}}(\mathbf{k})\right\}^{2}+\Delta_{\mathbf{k}}^{2}}
$$

and

$$
E_{\mathbf{k}}^{+}=\sqrt{\left\{\epsilon_{\mathbf{k}}-\mu+t_{\perp}^{\mathrm{eff}}(\mathbf{k})\right\}^{2}+\Delta_{\mathbf{k}}^{2}}
$$

and the gap parameter turns out to be

$$
\Delta_{\mathbf{k}}=\frac{\sum_{\mathbf{k}^{\prime}} \Delta_{\mathbf{k}^{\prime}} V_{\mathbf{k}, \mathbf{k}^{\prime}}\left(\chi\left(E_{\mathbf{k}}^{-}\right)+\chi\left(E_{\mathbf{k}}^{+}\right)\right) / 2}{1-T_{p}^{\mathrm{eff}}(\mathbf{k})\left(\chi\left(E_{\mathbf{k}}^{-}\right)+\chi\left(E_{\mathbf{k}}^{+}\right)\right) / 2}
$$

where $\chi\left(E_{\mathbf{k}}^{ \pm}\right)=\frac{1}{2 E_{\mathbf{k}}^{ \pm}} \tanh \left(\frac{\beta E_{\mathbf{k}}^{ \pm}}{2}\right)$. The pairing potential can be expanded as $V_{\mathbf{k}, \mathbf{k}^{\prime}}=$ $V \eta_{\mathbf{k}} \eta_{\mathbf{k}^{\prime}}$, where $\eta_{k}$ is the basis function of the $c_{4 v}$ point group. This makes it possible to write $\Delta_{\mathbf{k}}$ in terms of a $\mathbf{k}$-independent gap $\Delta$, the basis function $\eta_{\mathbf{k}}$ and the interaction parameter $V$. Finally, the self consistent equation for superconducting gap becomes

$$
\frac{1}{4 V}=\frac{1}{N} \sum_{\mathbf{k}} \frac{\eta_{\mathbf{k}}^{2}\left(\chi\left(E_{\mathbf{k}}^{-}\right)+\chi\left(E_{\mathbf{k}}^{+}\right)\right) / 2}{1-T_{p}^{\operatorname{eff}}(\mathbf{k})\left(\chi\left(E_{\mathbf{k}}^{-}\right)+\chi\left(E_{\mathbf{k}}^{+}\right)\right) / 2}
$$

and the expression for chemical potential is

$$
1-\delta=1-\frac{1}{N} \sum_{\mathbf{k}}\left(\epsilon_{\mathbf{k}}-\mu-t_{\perp}^{\mathrm{eff}}\right) \chi\left(E_{\mathbf{k}}^{-}\right)-\frac{1}{N} \sum_{\mathbf{k}}\left(\epsilon_{\mathbf{k}}-\mu+t_{\perp}^{\mathrm{eff}}\right) \chi\left(E_{\mathbf{k}}^{+}\right)
$$

Regarding the order parameter symmetry, though the issue is yet to be settled on a definite footing, a consensus seems emerging in the many experiments done on layered cuprates. The angle resolved photoemission spectroscopy (ARPES) measurements and 
other phase sensitive experiments indicate the $d_{x^{2}-y^{2}}$ symmetry of the order parameter [1], 2], and we consider the same in our calculations. This means the basis function $\eta_{\mathbf{k}}=\left(\cos k_{x} a-\cos k_{y} a\right) / 2$, which changes sign under $\pi / 2$ rotation. The interaction parameter $V$ is thus the nearest neighbour and attractive.

Bilayer Splitting: For bilayer cuprates, the presence of effective single particle hopping between the layers should be observable in the single particle density of states (DOS). In our calculations, the realistic six parameter dispersion for Bi2212, used to model the in-plane band structure, shows a power law singularity in the DOS in the form of a single peak. Inclusion of ISPH between the $\mathrm{CuO}_{2}$ layers would split the bands and the corresponding DOS will have two peaks. This phenomenon is known as bilayer splitting. However, no splitting is observed experimentally in the Bi2212 systems [19] even at low temperatures where broadening due to finite lifetime of quasiparticles is expected to be small. In Fig.1, we plot the DOS, $N(\xi)$ at two different temperatures where $\xi_{\mathbf{k}}=\epsilon_{\mathbf{k}} \pm t_{\perp}^{\mathrm{eff}}(\mathbf{k})$. Temperature dependence of $N(\xi)$ comes only through the probability factor $P$. Parameters for the plots are chosen to be $t_{\perp}=40 \mathrm{meV}$ and $E_{g}=8 \mathrm{meV}$, same as used to match the gap-variation data in Fig.3. At low temperatures $T=10 \mathrm{~K}$ and with no broadening (i.e. broadening parameter $\Gamma=0$ ), we find that $N(\xi)$ has two peaks (solid line) separated by an energy $\sim 3 \mathrm{meV}$. However, inclusion of a small broadening $\Gamma=3 \mathrm{meV}$ (dashed line) smears the two peaks and one broad peak appears. At a higher temperature $T=50 \mathrm{~K}$, the larger peak separation energy $\sim 21 \mathrm{meV}$ is due to the enhanced probability of the interlayer single particle hopping. Inclusion of nonzero $\Gamma$ (dashed lines) broadens the peaks and the value of $\Gamma$ needed for complete smearing of two peaks is $\Gamma \geq 10 \mathrm{meV}$. This is demonstrated in the lower panel ( $T=50 \mathrm{~K})$ of Fig.1.

It should be mentioned here that, the temperature dependence of the broadening parameter for layered cuprates is noted to follow [3] the relation $\Gamma(\mathrm{meV}) \approx \Gamma_{0}+$ $2.5 T_{c}\left(T / T_{c}\right)^{3}$, where $\Gamma_{0}$ is the zero temperature value. Although high values of $\Gamma_{0} \sim$ $15 \mathrm{meV}$ is observed for Bi-cuprates [3], in our case a $\Gamma_{0} \sim 7-8 \mathrm{meV}$ could be enough to remove the splitting at all temperatures. Moreover, at low temperatures $(T=10 \mathrm{~K})$ 
the peak separation is small $\sim 3 \mathrm{meV}$ (solid line for $T=10 \mathrm{~K}$ in Fig.1), whereas in the ARPES experiments the energy resolution is far beyond this level (resolution function FWHM $19 \mathrm{meV}$ ) 20 and the small peak separation would probably remain unresolved. Thus, in the cleanest sample and at very low temperature, even if one ideally takes $\Gamma_{0} \rightarrow 0$, the bilayer splitting in Bi-cuprates would remain unobservable by experiments. Finally, it has been noted that YBCO systems have a smaller $\Gamma_{0} \sim 1.5 \mathrm{meV}$ [3] and also have orthorhombic distortions, which might explain why splitting could possibly be observed in YBCO [21] in contrast to the Bi-cuprates.

So far, we have discussed the issue of bilayer splitting by calculating the normal state density of states. But, in actual ARPES experiments, bilayer splitting is addressed by studying the photoemission intensity curves in the superconducting state. Within the modified ILT model, we have calculated the photoemission intensity curves [11] in the superconducting state and found that, with an energy resolution much better than that in actual experiments 20] and with a very small boradening $\Gamma \sim 1 \mathrm{meV}$, bilayer splitting remains unobservable for temperatures ranging upto $50 \mathrm{~K}$. In other words, the inclusion of an effective ISPH in the modified ILT model is in perfect tune with the experimental observations.

Issues related to the Superconducting Gap: The superconducting gap and other quantities are calculated by simultaneous solutions of the self consistent $\operatorname{Eq}(7)$ and $\mathrm{Eq}(8)$ for the gap and the chemical potential respectively, implementing numerical techniques.

In our calculations, doping is kept fixed at the optimum level $\left(\delta_{\text {opt }}\right)$ at which $T_{c}$ attains its maximum value $T_{c}^{m}$. Position of $\delta_{\text {opt }}$ is found to have a slow dependence on the value of $E_{g}$ considered. A study of $T_{c}$ versus $\delta$, for different $E_{g}$, shows that with $E_{g} \leq 5 \mathrm{meV}, T_{c}$ has a two-peak structure, whereas for higher $E_{g}$ a one-peak bellshaped form is recovered consistent with that in layered cuprates. Thus, we enforce the condition $E_{g}>5 \mathrm{meV}$. Value of bare single particle hopping matrix element is taken to be $t_{\perp}=40 \mathrm{meV}$ in accordance with the band structure calculations suggesting $t_{\perp} /\left|t_{1}\right| \sim$ $0.2-0.3$. Interaction parameter is taken to be $V=80 \mathrm{meV}$ which makes $T_{c}^{m} \sim 100 \mathrm{~K}$ 
for $E_{g} \sim T_{c}^{m}$. This value of $V$ is kept fixed throughout in this communication.

In Fig.2(a) we plot $T_{c}^{m}$ as a function of $t_{\perp}$. Different curves, labelled by capital alphabets, correspond to various $E_{g}$ values as, A:6, B:10, C:15 and D:20 in meV. Clearly, variation of $T_{c}^{m}$ with $t_{\perp}$ may take different shapes depending on the values of $E_{g}$. For small $E_{g}$, the suppression of coherent single particle hopping between the layers is weak and $T_{c}^{m}$ decreases with $t_{\perp}$, whereas large $E_{g}$ makes ISPH less probable and in turn the pair tunneling probability gets enhanced, which results a rise in $T_{c}^{m}$ with $t_{\perp}$. In the Anderson limit $\left(E_{g}=\infty\right), T_{c}^{m}$ grows rapidly with $t_{\perp}$.

Application of pressure on a sample along $c$-axis could reduce the out-of-plane lattice constant which results in an increase of the interlayer coupling. In the plot of $T_{c}^{m}$ versus $t_{\perp}$ in Fig.2(a), we find that, for large $E_{g}$ (underdoped materials), $T_{c}^{m}$ increases with $t_{\perp}$, for small $E_{g}$ (overdoped materials), $T_{c}^{m}$ decreases with $t_{\perp}$ and at some intermediate $E_{g}$, $T_{c}^{m}$ remains unchanged. Thus, within our model, $c$-axis applied pressure would result, an increase of $T_{c}$ for underdoping, a decrease of $T_{c}$ for overdoping and an unaffected $T_{c}$ for some intermediate doping. Similar varied behaviours of $T_{c}$ are seen for differently doped cuprates under hydrostatic or uniaxial pressure [22]. However, it may be mentioned that hydrostatic pressure could also inflict changes in the in plane parameters. Hence, we leave this result of various $T_{c}^{m}$ variation with $t_{\perp}$ as a prediction which could be checked by further experiments.

Variation of the zero temperature superconducting gap $\left(\Delta_{\mathrm{k}}^{\max }(0)\right)$ as a function of $t_{\perp}$ is shown in Fig.2(b). The $E_{g}$ values corresponding to different curves are same as used in Fig.2(a). Here, irrespective of the values of $E_{g}, \Delta_{\mathbf{k}}^{\max }(0)$ increases with $t_{\perp}$ in the same fashion for every curve.

A plot of $T_{c}^{m}$ as a function of $E_{g}$, for different $t_{\perp}$, is given in Fig.3. As is obvious, $T_{c}^{m}$ increases with increasing $E_{g}$ due to the progressive enhancement of effective pair tunneling probability, caused by $E_{g}$. As is seen, at some $E_{g}$ close to $\sim 12 \mathrm{meV}, T_{c}^{m}$ for all the curves are same, implying that the transition temperature is independent of $t_{\perp}$. 
In fact, we crosscheck that, $T_{c}^{m}$ as a function of $t_{\perp}$, for $E_{g} \approx 12 \mathrm{mev}$, is nearly flat.

Next, we come to the issue of the temperature dependence of the $d$-wave superconducting gap. A plot of scaled supercondcting gap $\Delta_{\mathbf{k}}^{\max }(T) / \Delta_{\mathbf{k}}^{\max }(0)$ as a function of reduced temperature $T / T_{c}$ is given in Fig.4. Solid line is the BCS-form of gapvariation and solid squares are experimental data on Bi-cuprate [4. Except at the edges $\left(T / T_{c}=0 \& 1\right)$ the locus of the experimental data is well below the BCS-curve and the absence of low temperature saturation in the experimental data stands out as an anomalous characteristic of high- $T_{c}$ cuprates. The dashed lines are from our calculations, but, the one very close to the BCS-curve represents the gap variation for the original ILT model $\left(E_{g}=\infty\right)$. The dashed curve with $E_{g}=8 \mathrm{meV}$ is within the modified ILT model which matches experimental data very well. To the best of our knowledge, this is the first ever calculations where precise matching of experimental gap-variation data is obtained. An important and related quantity is the ratio of the zero temperature superconducting gap to $T_{c}$. In experiments on layered high- $T_{c}$ cuprates, high values of $2 \Delta_{\mathrm{k}}^{\max }(0) / T_{c} \sim 5-7$ is observed [3], compared to the BCS superconductors where it is $\sim 3.5$. In the inset of Fig. 4 we plot the gap-ratio as a function of $t_{\perp}$ for the same parameters used to fit the experimental gap-variation data. The gap-ratio increases with $t_{\perp}$ and within the realistic range of $t_{\perp}(\sim 30-45 \mathrm{meV})$ we recover the ratio $\sim 5-7$. To be precise, at $t_{\perp}=40 \mathrm{meV}$, we get $2 \Delta_{\mathbf{k}}^{\max }(0) / T_{c}=5.8$. Note that, for same parameters and with $E_{g}=\infty$ (Anderson limit), we find $2 \Delta_{\mathrm{k}}^{\max }(0) / T_{c}=4.4$. High value of the gapratio and its increasing trend with $t_{\perp}$ within the modified model, could be understood as follows.

For any finite $E_{g}$, when $T \rightarrow 0$, the probability factor $P \rightarrow 0$, ISPH is completely blocked and $\Delta_{\mathbf{k}}^{\max }(0)$ grows with $t_{\perp}$ since $t_{\perp}$ acts only to enhance $T_{p}^{\text {eff }}$. But, for any finite $T>0$ the probability factor $P \neq 0$, blocking of the effective ISPH is not complete and one gets a transition temperature $T_{c}(P \neq 0)<T_{c}(P=0)$ resulting in a high value of the gap-ratio. Regarding the increasing trend of gap-ratio, consider the situation when $T_{c}^{m}$ decreases or saturates with $t_{\perp}$ for small $E_{g}$ (as in Fig.2(a)). But notice that, $\Delta_{\mathbf{k}}^{\max }(0)$ 
always rises with $t_{\perp}$ (see Fig.2(b)) yielding the observed result of the gap-ratio. Even for the case when $T_{c}$ increases with $t_{\perp}$ (for high $E_{g}$ ), note that its increase is slower than $\Delta_{\mathbf{k}}^{\max }(0)$ which effectively increases the gap-ratio with $t_{\perp}$. For a fixed value of $t_{\perp}$, one gets an increasing gap-ratio with decreasing $E_{g}$ which could also follow from similar arguments.

To conclude, we have considered a modified interlayer pair tunneling model where pair tunneling is accompanied by interlayer single particle hopping. Within the model, absence of bilayer splitting is established corresponding to experimental situation. Different types of variation of $T_{c}$ as a function of interlayer coupling, for different values of the pseudogap, comes out as a prediction that corresponds to $T_{c}$ variation under $c$-axis pressure. Temperature dependent superconducting gap from our calculations correctly reproduces the experimental data, and for realistic parameters, high values of the gap-ratio are obtained. 


\section{References}

[1] W. N. Hardy et al., Phys. Rev. Lett. 70, 3999 (1993); D. A. Brawner and H. R. Ott, Phys. Rev. B 50, 6530 (1994); J. R. Kirtley et al., Nature (London) 373, 225 (1995).

[2] Z. X. Shen et al., Phys. Rev. Lett. 70, 1553 (1993); H. Ding et al., Phys. Rev. B 54, 9678 (1996).

[3] T. Hasegawa, H. Ikuta and K. Kitazawa, in Physical Properties Of High Temperature Superconductors, Vol.III, ed. D. M. Ginsberg (World Scientific, 1992).

[4] M. Itoh, S. Karimoto, K. Namekawa and M. Suzuki, Phys. Rev. B 55, 12001 (1997).

[5] P. W. Anderson, in Superconductivity, Proceedings of the ICTP spring college in 1992, eds. P. Butcher and Y. Lu (World Scientific, Singapore).

[6] S. Chakravarty, A. Sudbo, P. W. Anderson and S. Strong, Science 261, 337 (1993).

[7] O. K. Andersen, A. I. Liechtenstein, O. Jepsen and F. Paulsen, J. Phys. Chem. Solids 56, 1573 (1995).

[8] S. L. Cooper and K. E. Gray, in Physical Properties of High Temperature Superconductors, Vol.IV, ed. D. M. Ginsberg (World Scientific, 1994).

[9] S. Uchida, K. Tamasaku and S. Tajima, Phys. Rev. B 53, 14558 (1996) and refrences therein.

[10] A. N. Das and S. Sil, Physica C (in press).

[11] B. Chattopadhyay and A. N. Das (unpublished).

[12] P. W. Anderson , Science 256 (1992) 1526; A. Sudbo, S. Chakravarty, S. Strong and P. W. Anderson, Phys. Rev. B 49, 12245 (1993); S. Chakravarty and P. W. Anderson, Phys. Rev. Lett. 72, 3859 (1994). 
[13] R. Fehrenbacher and M. R. Norman, Phys. Rev. Lett. 74, 3884 (1995); M. R. Norman et al., Phys. Rev. B 52, 615 (1995).

[14] B. Chattopadhyay, D. Gaitonde and A. Taraphder, Europhys. Lett. 34, 705 (1996); B. Chattopadhyay, Phys. Lett. A 226, 231 (1997); B. Chattopadhyay, J. Lahiri and A. N. Das, Mod. Phys. Lett. B 11, 1285 (1997).

[15] D. M. King et al., Phys. Rev. Lett. 73, 3298 (1994); Z. X. Shen et al., Science 267, 343 (1995).

[16] A. G. Loeser et al., Science 273, 325 (1996); H. Ding et al., Nature 382, 512 (1996),

[17] B. Batlogg, in High Temperature Superconductivity, eds. K. S. Bedell et al. (Addison-Wesley, 1990); K. Tamasaku, Y. Nakamura and S. Uchida, Phys. Rev. Lett. 69, 1455 (1992).

[18] G. V. M. Williams et al., Phys. Rev. Lett. 78, 721 (1997).

[19] H. Ding et al., Phys. Rev. Lett. 76, 1533 (1996).

[20] M. R. Norman et al., Phys. Rev. B 76, 1533 (1996).

[21] J. C. Campuzano et al., Phys. Rev. Lett. 64, 2308 (1990); R. Liu et al., Phys. Rev. B 46, 11056 (1992); K. Gofron et al., Phys. Rev. Lett. 73, 3302 (1994).

[22] J. S. Schilling and S. Klotz, in Physical Properties Of High Temperature Superconductors, Vol.III, ed. D. M. Ginsberg (World Scientific, 1992); U. Welp et al., Phys. Rev. Lett. 69, 2130 (1992); L. Gao et al., Phys. Rev. B 50, 4260 (1994); J. G. Lin et al., Phys. Rev. B 53, 11855 (1996); D. Tristan et al., Phys. Rev. B 55, 11832 (1997). 


\section{Figure captions:}

Fig.1. Single particle density of states $N(\xi)$ is plotted at two different temperatures $T=10 K$ and $T=50 K$. Values of broadening parameter $\Gamma$ used are given in the figures. The full bandwidth of energy $\xi$ is not shown, since the peaks in the DOS are only of concern for the purpose of addressing the issue of bilayer splitting.

Fig.2. (a) Mean-field transition temperature $\left(T_{c}^{m}\right)$ at optimal doping as a function of bare interlayer coupling $t_{\perp}$ for different $E_{g}$ represented by alphabetic labels as A:6, B:10, $\mathrm{C}: 15, \mathrm{D}: 20$ in $\mathrm{meV}$. The interaction parameter is $V=80 \mathrm{meV}$.

(b) A plot of the maximum value of the zero temperature superconducting gap. Values of $E_{g}$ for different curves are as shown and the alphabetic labels corresponds to same $E_{g}$ values as in (a).

Fig.3. Transition temperature as a function of the normal state pseudogap magnitude $E_{g}$ for three different values of $t_{\perp}$, as shown in the figure. Doping is set to the optimum level (for $E_{g}=8 \mathrm{meV}$ ) and $V=80 \mathrm{meV}$.

Fig.4. Temperature variation of the scaled superconducting gap $\left(\Delta_{\mathbf{k}}^{\max }(T) / \Delta_{\mathbf{k}}^{\max }(0)\right)$ as a function of the reduced temperature $\left(T / T_{c}\right)$ is presented. Solid line corresponds to the conventional BCS superconductors and solid squares are experimental data from Ref. 4 . Dashed lines are from our calculations for different $E_{g}$ shown. [Inset: A plot of the ratio $\Delta_{\mathbf{k}}^{\max }(0) / T_{c}$ as a function of $t_{\perp}$. The parameters are $V=80 \mathrm{meV}$ and $E_{g}=8 \mathrm{meV}$ that yielded match to the experimental gap-variation data as in the main figure.] 


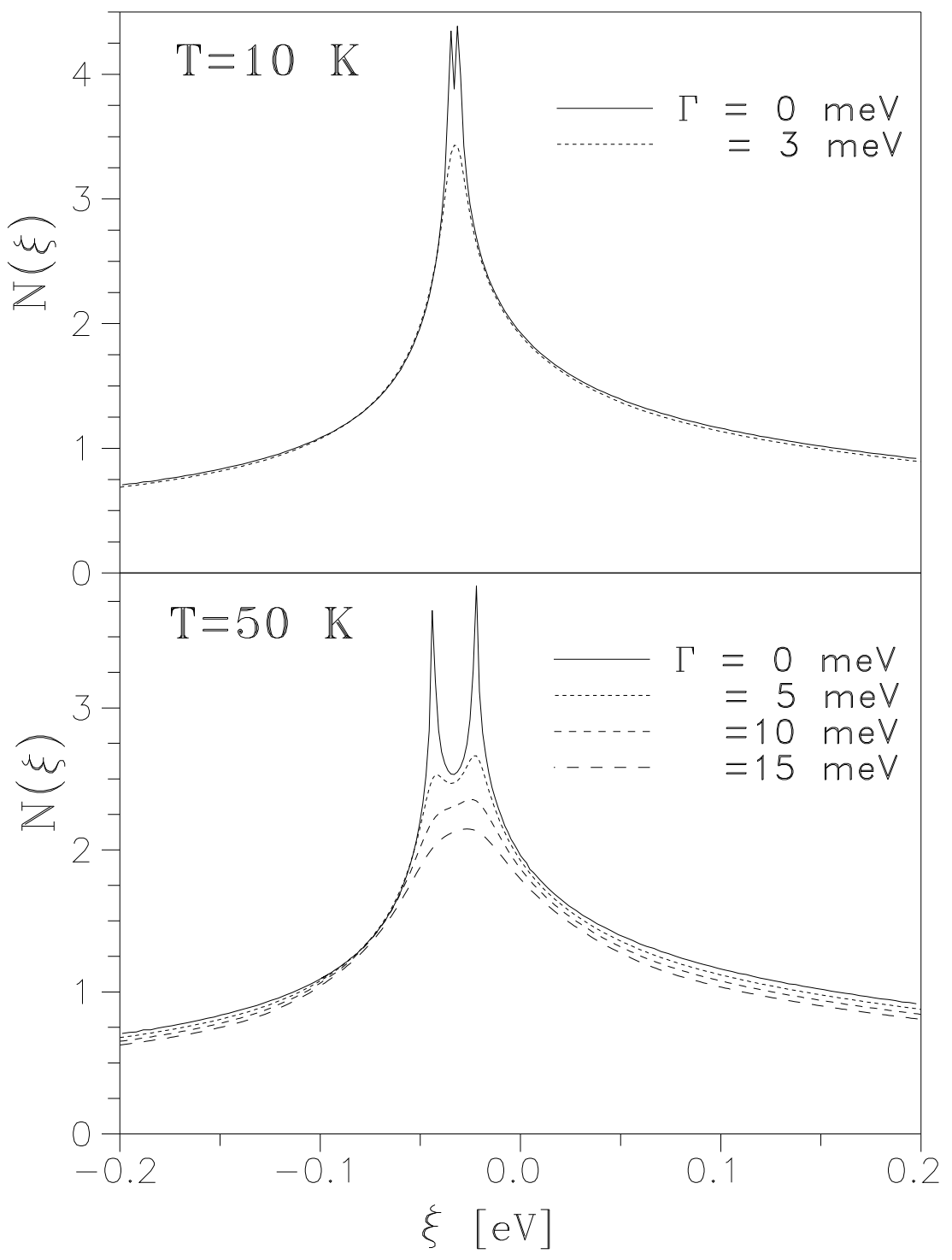




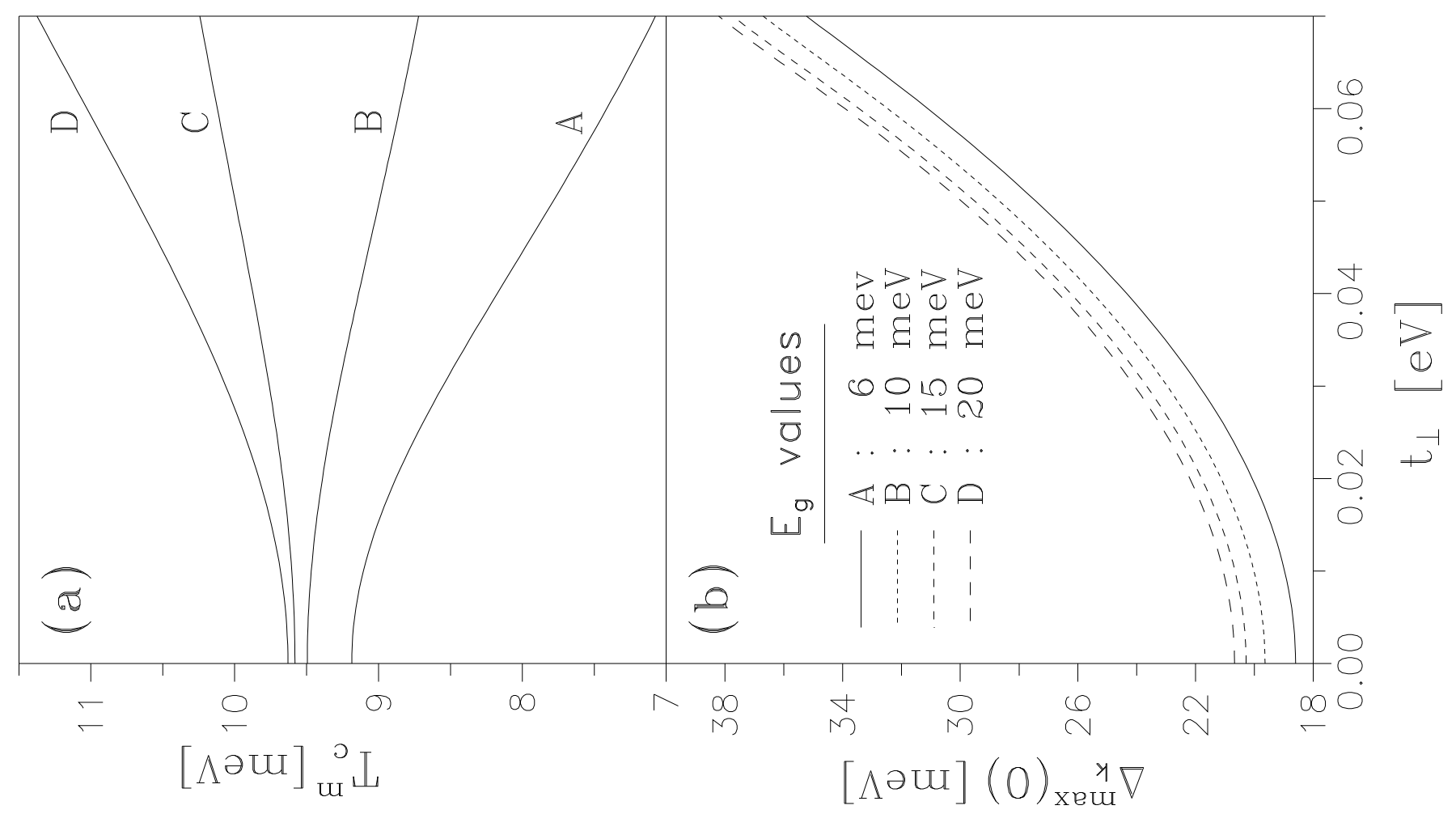




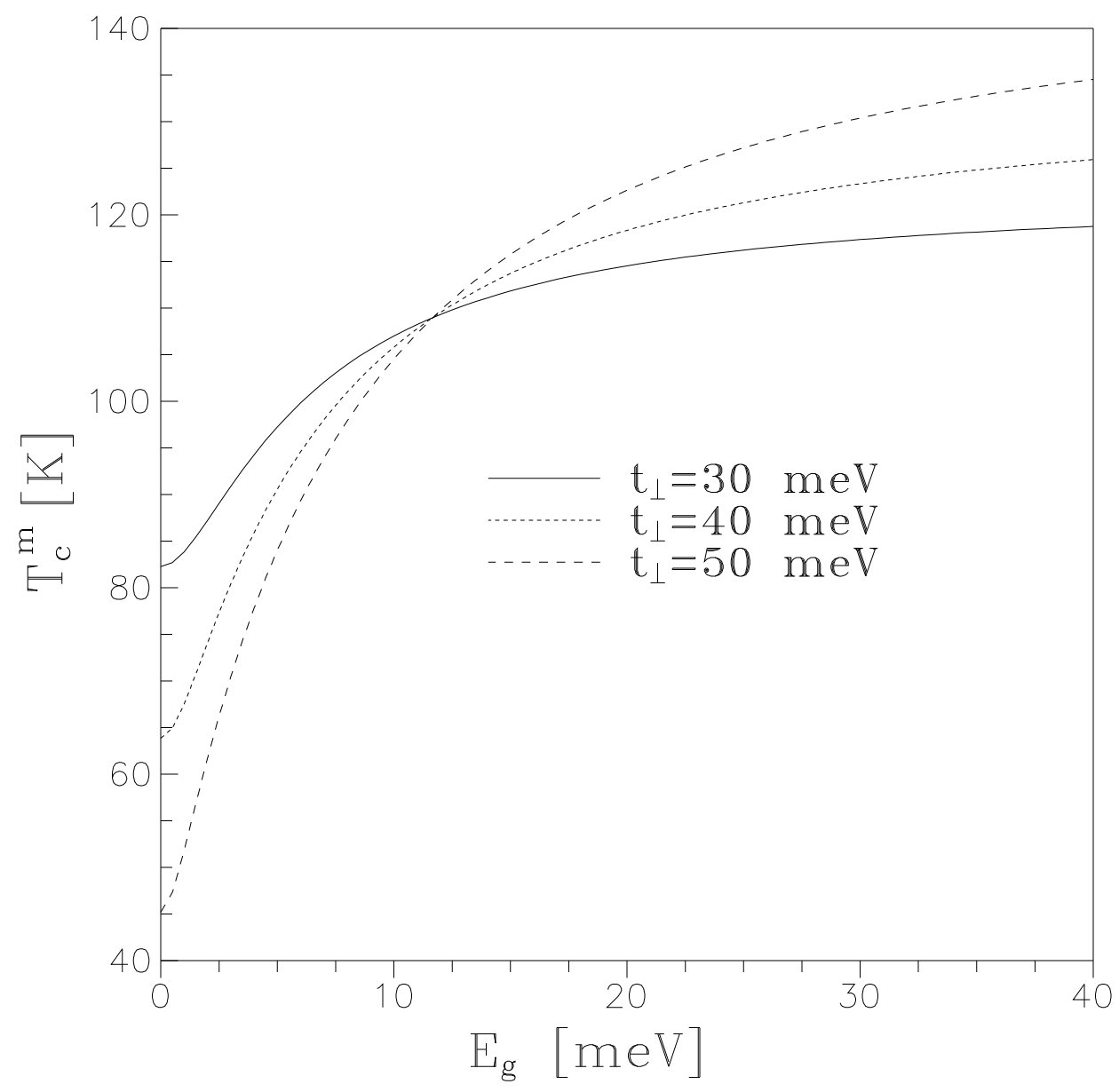




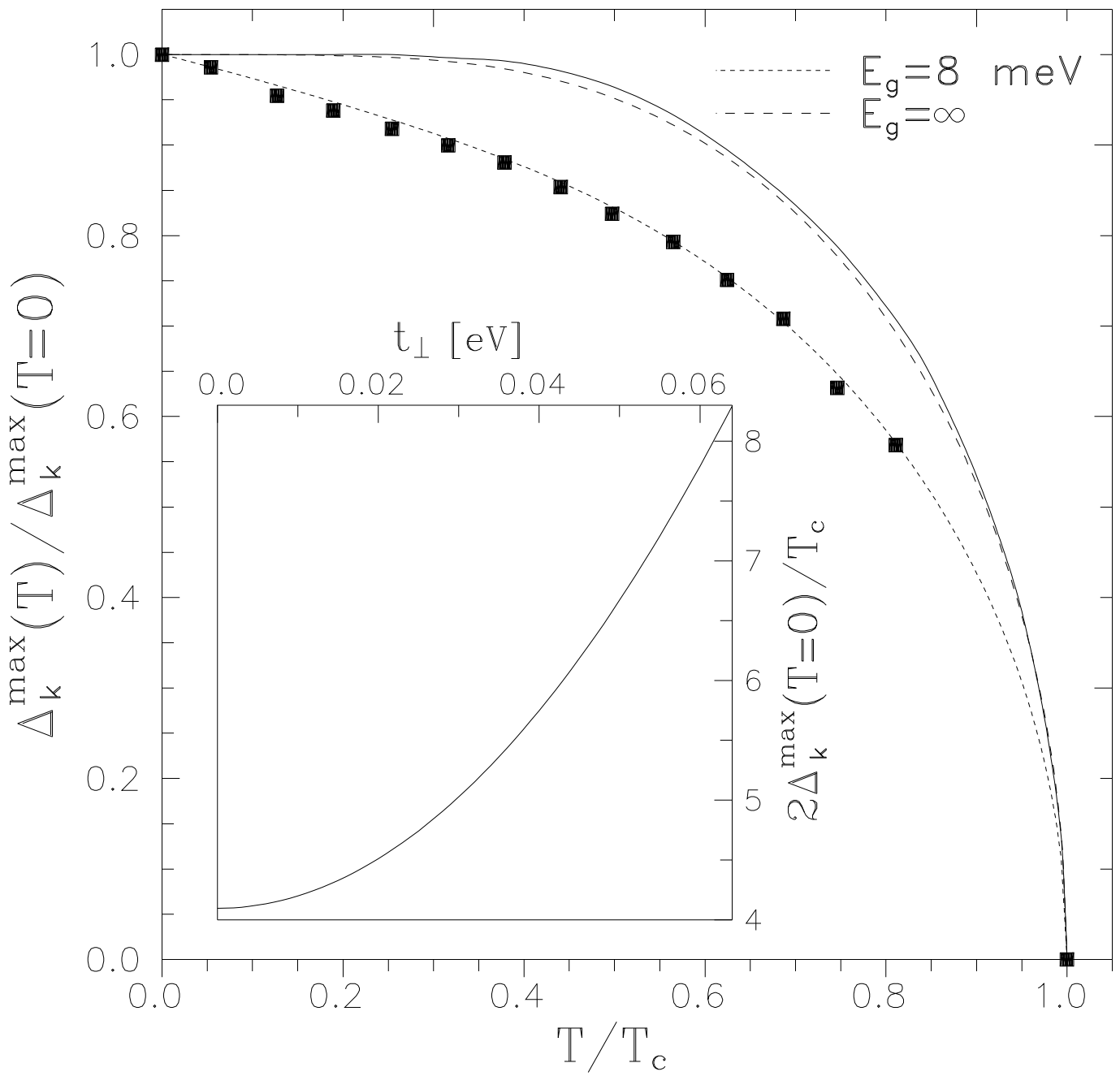

